\title{
The Universal Partition Theorem for Oriented Matroids*
}

\author{
H. Günzel \\ Department of Mathematics (C), \\ Aachen University of Technology, \\ D-52056 Aachen, Germany
}

\begin{abstract}
We consider realization spaces of a family of oriented matroids of rank three as point configurations in the affine plane. The fundamental problem arises as to which way these realization spaces partition their embedding space. The Universal Partition Theorem roughly states that such a partition can be as complicated as any partition of $\mathbb{R}^{n}$ into elementary semialgebraic sets induced by an arbitrary finite set of polynomials in $\mathbb{Z}[X]$. We present the first proof of the Universal Partition Theorem. In particular, it includes the first complete proof of the so-called Universality Theorem.
\end{abstract}

\section{Introduction}

We study the question of which way realization spaces of oriented matroids partition their embedding space, the $n$-dimensional Euclidean space $\mathbb{R}^{n}$. We compare the latter partitions with partitions of $\mathbb{R}^{n}$ into elementary semialgebraic sets defined by a finite family of polynomials with entire coefficients. For any partition defined by such a family of polynomials we construct a family of oriented matroids inducing the same partition (up to a product with a smooth manifold $N$ ). This is (roughly) the assertion of the Universal Partition Theorem.

This result-even a stronger one-has been stated earlier by Mnëv [5]. At the end of this section we compare the earlier statement and the present one. Before this, we summarize what is known about the proof. Simultaneously, we point out the contribution made here and give an outline of how it works.

At the present time a proof of the Universal Partition Theorem does not seem to be available. However, there is a sketch of a proof for the Universality Theorem which is implied by the Universal Partition Theorem. The latter theorem is due to Mnëv and

\footnotetext{
* This work was supported by the Deutsche Forschungsgemeinschaft, Graduiertenkolleg "Analyse und Konstruktion in der Mathematik."
} 
compares one element from each of the above partitions. It states that for any (specific) elementary semialgebraic set defined over $\mathbb{Z}$ a diffeomorphic realization space of an oriented matroid (up to a product with a smooth manifold $N$ ) exists. (Here we use the restriction of a smooth embedding to the set under consideration.) We emphasize that the Universal Partition Theorem is stronger than the Universality Theorem in the sense that it even shows the complexity of fitting together the realization spaces.

Generally speaking, Mnëv's approach works as follows (see [4] and also [6]). First, complicated polynomials can be obtained from $\mathbb{Z}[X]$ by means of elementary operations (such as addition and multiplication). This yields a finite cascade of such operations starting with $-1, X_{1}, X_{2}, \ldots, X_{n}$ which generates the polynomials inducing our semialgebraic partition of $\mathbb{R}^{n}$. Second, arithmetical operations can be realized geometrically as point configurations in $\mathbb{R}^{2}$. This can be done by means of the so-called von Staudt constructions [7]. This implies the geometrical computation of our polynomials. The latter computation works roughly as follows. We use a vector in $\mathbb{R}^{n}$ as the input $x$. The latter corresponds to a point $P^{i}\left(x_{i}\right)$ of our configuration. The cascade of elementary geometrical computation carried out generates a configuration. The locus of all such configurations for input $x$ from a specific elementary semialgebraic set is the corresponding realization space. The main problem is to keep the underlying oriented matroid fixed as $x$ varies within the given set.

In Mnëv's sketch the points $b \in N$ play the role of (control) parameters. Assume that $N$ is embedded into $\mathbb{R}^{k}$, then, for any point from our elementary semialgebraic set, the values $0<b_{1}<b_{2}<\cdots<b_{k}$ are chosen appropriately. However, finally, the following two questions are not answered satisfactorily in the argumentation performed in [4]:

(1) Is it true that the same oriented matroid $\mathcal{M}$ can be realized whenever $b_{i+1} / b_{i}$ is large enough?

(2) Assume that (1) is answered affirmatively; how can the product structure of the realization space be proved? Are the spaces of "good choices" for $b_{1}, \ldots, b_{k}$ (realizing $\mathcal{M}$ ) diffeomorphic for all input points from our elementary semialgebraic set?

The key idea in our proof is the introduction of supporting points for our computations generating the so-called computation frame. Given the computation frame, the input $x$ defines the whole geometrical computation in a unique way. The admissible frames are given by means of the underlying oriented matroid. The main point is that such a computation frame can be chosen in a regular way. This gives rise to the concept of a regular computation frame. If we use a regular computation frame, then we are able to control the relative position of all points of our configuration with respect to any line connecting two of them. More precisely, the oriented matroid of a computation using the input $x$ can be computed from the oriented matroid of the frame used and the sign-code of the elementary semialgebraic set containing $x$. The concept of a regular computation frame is the main tool in our proof of the Universal Partition Theorem.

The idea of a geometrical computation of polynomial systems has been developed independently from Mnëv by Bokowski and Sturmfels [2]. However, they do not control the underlying (oriented) matroid. This yields a weaker theorem, replacing "diffeomorphic" by "birationally isomorphic." We emphasize that the original statements of the 
Universal Partition Theorem and the Universality Theorem [4], [5] go beyond the scope of the results proven here in the following main points; however, recall that at present no (complete) proof of these results is available:

(1) $N$ could be of trivial topology.

(2) Open semialgebraic sets correspond to uniform oriented matroids.

Assertion (1) provides a complete picture of possible homotopy types for realization spaces of oriented matroids, whereas our theorem gives an estimation of the smallest complexity that can be obtained. For more discussion see Section 7. Assertion (2) could also be achieved in our setting-for the Universality Theorem. To this end we would have to generate a constructible oriented matroid, which would complicate our construction a little.

The paper is organized as follows. In Section 2 we briefly introduce the concepts of an oriented matroid and its realization space. Then we formulate the Universal Partition Theorem. Elements from projective geometry which are necessary to understand our geometrical models for arithmetical operations are summarized in Section 3. In Section 4 we realize geometrically the cascade of elementary operations generating our defining functions. We also define the computation frame. Section 5 contains a proof of the Universal Partition Theorem for regular computation frames. In Section 6 we finally prove the existence of a regular computation frame, thus completing the proof of the Universal Partition Theorem.

\section{The Universal Partition Theorem}

In this section we introduce the concept of an oriented matroid over a basic set $\mathcal{X}=$ $\{1, \ldots, k\}$ by means of signed circuits [1]. There are different models for oriented matroids, each inducing a concept of a realization space. For our purposes it is convenient to consider realizations as point configurations (in $\mathbb{R}^{2}$ ). At the end of this section we formulate the Universal Partition Theorem.

\section{Notation (used in Definition 1).}

$$
\begin{aligned}
& 2^{\mathcal{X}} \text { Power set of } \mathcal{X} . \\
& 2_{*}^{\mathcal{X}} 2^{\mathcal{X}} \backslash\{\emptyset\} . \\
&\left(X^{+}, X^{-}\right) \text {For } X \in 2^{\mathcal{X}} \times 2^{\mathcal{X}} \text { put } X=\left(X^{+}, X^{-}\right) \text {and define }-X:=\left(X^{-}, X^{+}\right) \\
& \leq \quad \text { For } X, X_{2} \in 2^{\mathcal{X}} \times 2^{\mathcal{X}} \text { we define: } \\
& X_{1} \leq X_{2} \Leftrightarrow \Leftrightarrow X_{1}^{+} \subset X_{2}^{+} \text {and } X_{1}^{-} \subset X_{2}^{-} \\
& \sup \quad \text { For } \mathcal{Y} \subset 2^{\mathcal{X}} \times 2^{\mathcal{X}} \text { let } \sup \mathcal{Y}:=\left(\bigcup_{Y \in \mathcal{Y}} Y^{+}, \bigcup_{Y \in \mathcal{Y}} Y^{-}\right) .
\end{aligned}
$$

Definition 1. Let $\mathcal{C}$ be a subset of $2_{*}^{\mathcal{X}} \times 2_{*}^{\mathcal{X}}$ such that the following axioms are satisfied:

- $X \in C \Rightarrow-X \in C$ (Symmetry).

- $X_{1}, X_{2} \in \mathcal{C}, X_{1} \leq X_{2} \Rightarrow X_{1}=X_{2}$ (Minimality).

- $X_{1}, X_{2} \in C, X_{2} \neq-X_{1}, x \in X_{1}^{+} \cap X_{2}^{-} \Rightarrow \exists X_{3} \in C: X_{3} \leq \sup \left\{X_{1}, X_{2}\right\}$, and $x \notin X_{3}^{+} \cup X_{3}^{-}$(Exchange of the basis). 
Then $\mathcal{M}:=(\mathcal{X}, C)$ is called an orientation matroid (over basic set $\mathcal{X}$ ) and the elements of $\mathcal{C}$ are called signed circuits. The length of a signed circuit $X \in \mathcal{C}$ is defined as $|X|:=$ $\left|X^{+} \cup X^{-}\right|$. The numbers $|\mathcal{M}|:=|\mathcal{X}|$ and $\operatorname{rank}(\mathcal{M}):=\min \left\{|\mathcal{M}|, \max _{X \in \mathcal{C}}|X|-1\right\}$ are referred to as the cardinality and the rank of $\mathcal{M}$, respectively.

Remark 2. Consider the $k$-tuple $P=\left(P_{1}, \ldots, P_{k}\right)$ of points in $\mathbb{R}^{2}$. Put $\mathcal{X}:=\{1, \ldots, k\}$. Then $X \in 2_{*}^{\mathcal{X}} \times 2_{*}^{\mathcal{X}}$ is called a signed circuit if it is minimal with the property that the convex hulls $\operatorname{conv}\left\{P_{i} \mid i \in X^{+}\right\}$and $\operatorname{conv}\left\{P_{j} \mid j \in X^{-}\right\}$have a nonempty intersection. A moment of reflection shows that the set $\mathcal{C}(P)$ of signed circuits defines an oriented matroid structure, $\mathcal{M}(P)$, on $\mathcal{X}$.

Let $\mathcal{M}_{3}(k)$ denote the set of rank-three oriented matroids with cardinality $k$. For $\mathcal{M} \in \mathcal{M}_{3}(k)$ the set $\mathcal{R}(\mathcal{M}):=\left\{P \in\left(\mathbb{R}^{2}\right)^{k} \mid \mathcal{M}(P)=\mathcal{M}\right\}$ is called the realization space of $\mathcal{M}$. Thus, the space $\mathcal{R}(\mathcal{M})$ is the locus of all point configurations in $\mathbb{R}^{2}$ with the underlying oriented matroid $\mathcal{M}$. Note that the union of the realization spaces belonging to $\mathcal{M}_{3}(k)$ forms an open and dense subset of $\left(\mathbb{R}^{2}\right)^{k}$.

The following theorem says that there are families of rank-three oriented matroids such that although their realization spaces fit together forming a smooth manifold (as their union), this fitting can be almost arbitrarily bad.

Theorem (Universal Partition Theorem). Let $f_{1}, \ldots, f_{m} \in \mathbb{Z}\left[X_{1}, \ldots, X_{n}\right]$. For any sign-vector $\sigma \in\{-1,0,1\}^{m}$ let

$$
M_{\sigma}:=\left\{x \in \mathbb{R}^{n} \mid \operatorname{sign} f_{i}(x)=\sigma_{i}\right\}
$$

denote the corresponding elementary semialgebraic set. Then there is an injective mapping $\psi$ assigning to any sign-vector $\sigma \in\{-1,0,1\}^{m}$ a rank-three oriented matroid $\mathcal{M}_{\sigma} \in \mathcal{M}_{3}(k)$ (with fixed $k$ ), and there are a smooth manifold $N \neq \emptyset$ and a smooth embedding $\varphi: \mathbb{R}^{n} \times N \hookrightarrow\left(\mathbb{R}^{2}\right)^{k}$ such that the following relation (*) holds for all sign-vectors:

$$
\mathcal{R}\left(\mathcal{M}_{\sigma}\right)=\varphi\left(M_{\sigma} \times N\right)
$$

Here, sign $y$ is defined as $-1,0$, and 1 for negative, zero, and positive values of $y \in \mathbb{R}$, respectively.

Remark 3. In fact, any (elementary) semialgebraic set $M_{\sigma}$ coincides with the corresponding realization space $\mathcal{R}\left(\mathcal{M}_{\sigma}\right)$ up to the product with the smooth manifold $N$ and application of the smooth embedding $\varphi$ into the common embedding space $\left(\mathbb{R}^{2}\right)^{k}$ of rank-three oriented matroids with cardinality $k$.

\section{Elements of Projective Geometry}

In this section we summarize the background material from projective geometry that is needed in our construction. The assertions contained in this section (except Lemma 10) could also have been taken or easily derived from textbooks on projective geometry, 
such as [7]. We present this short introduction for two reasons. First, we have to explain our specific geometrical models for arithmetical operations, which differ from ones presented in the textbooks. The use of these models is motivated by the requirement to have a constant oriented matroid for all the input data the models are used for. Second, we obtain a completely self-contained presentation.

Let $G(3,1)$ denote the Grassmann manifold of one-dimensional linear subspaces of the Euclidean space $\mathbb{R}^{3}$. The manifold $\mathbb{P}^{2}=G(3,1)$ is also called the projective plane. Let $\langle\cdot, \cdot\rangle$ denote the corresponding inner product. There is a natural way of defining a chart of $G(3,1)$ by means of an affine subspace $A_{v}:=\left\{x \in \mathbb{R}^{3} \mid\langle x, v\rangle=1\right\}$ with nonvanishing $v \in \mathbb{R}^{3}$ :

$$
\begin{array}{lll}
\varphi_{v}: U_{v} \rightarrow A_{v}=\mathbb{R}^{2}, & \text { where } U_{v}:=\{L \in G(3,1) \mid L \not L v\}, \\
\varphi_{v}: L \mapsto x /\langle x, v\rangle, & \text { where } L=\mathbb{R} x .
\end{array}
$$

Here, $L \perp v$ abbreviates orthogonality.

Now, a plane $P$ in $\mathbb{R}^{3}$ (a two-dimensional linear subspace) can be identified with the set of its one-dimensional subspaces $L_{P}:=\{L \in G(3,1) \mid L \subset P\}$. The set $L_{P}$ is called

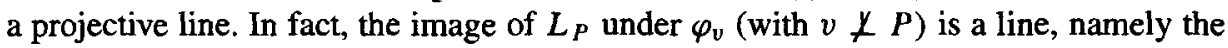
intersection $A_{v} \cap P$. For each chart $\varphi_{v}$ there is precisely one projective line $L_{v}^{\infty}$ which has more than one point outside $U_{v}$. This line corresponds to the plane in $\mathbb{R}^{3}$ which is orthogonal to $v$, i.e., it does not even meet $U_{v}$. It is called the line at infinity with respect to chart $\varphi_{v}$. Including the line at infinity we see that the projective lines are in one-to-one correspondence with their images under a (fixed) chart.

Two different projective lines have excactly one common point. In particular, any projective line intersecting $U_{v}$ has exactly one (projective) point in common with $L_{v}^{\infty}$. Moreover, the intersection point of two different projective lines meeting $U_{v}$ lies in $L_{v}^{\infty}$ if and only if they appear parallel in chart $\varphi_{v}$. We deduce that a family of lines is parallel (in chart $\varphi_{v}$ ) if and only if they have a common intersection point with $L_{v}^{\infty}$. Moreover, for any projective line $L$ there is a chart with $L=L_{v}^{\infty}$.

For the definition of geometrical computations we use scales on projective lines. They are defined in the following way. Let $L \subset G(3,1)$ be a projective line and assume three distinct points on it, say $O_{L}, 1_{L}$, and $\infty_{L}$. We choose a chart $\varphi_{v}$ with $\infty_{L} \in L_{v}^{\infty}$, i.e., $v \perp \infty_{L}$. Let $\psi: \varphi(L) \rightarrow \mathbb{R}$ denote the affine isomorphism defined by $\psi: \varphi_{v}\left(0_{L}\right) \mapsto 0$ and $\varphi_{v}\left(1_{L}\right) \mapsto 1$. Then $\omega:=\psi \circ \varphi_{v}$ extended by $\infty_{L} \mapsto \infty$ is called a projective scale $\omega: L \rightarrow \overline{\mathbb{R}}$. Here $\overline{\mathbb{R}}$ stands for the one-point compactification of $\mathbb{R}$. Until now, we do not know whether or not a projective scale also depends on the particular choice of $v$. The projective scales defined for multiples of $v$ obviously coincide. We say that such charts form a family. For different families of charts $\varphi_{v^{1}}, \varphi_{v^{2}}\left(v^{i} \perp \infty_{L}\right)$ we can choose special values for $v_{1}$ and $v_{2}$ such that $A_{v^{1}} \cap P_{L}=A_{v^{2}} \cap P_{L}$, where $P_{L}$ denotes the plane in $\mathbb{R}^{3}$ defining $L$. This implies $\varphi_{v_{1}}(L)=\varphi_{v_{2}}(L)$, i.e., $\omega$ is uniquely defined by the choice of $O_{L}, 1_{L}$, and $\infty_{L}$. Note that projective scales are defined for lines in the affine plane, too. This is possible since the affine plane can be considered as a chart of the projective plane.

Next, we consider projective isomorphisms and their influence on projective scales. Any linear isomorphism $\mathbb{R}^{3} \rightarrow \mathbb{R}^{3}$ induces a diffeomorphism $G(3,1) \rightarrow G(3,1)$. The latter diffeomorphism is called a projective isomorphism. Chosing charts compatible 


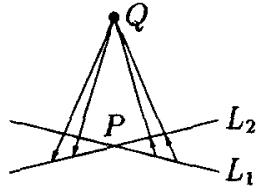

(a)

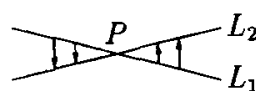

(b)

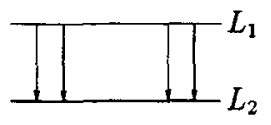

(c)

Fig. 1

with a linear isomorphism, we have the following assertion concerning the associated projective isomorphism.

Lemma 4 (Connection Lemma). Let $\psi$ be a projective isomorphism on $\mathbb{P}^{2}$ and let $L_{1} \subset \mathbb{P}^{2}$ be a line equipped with a projective scale $\omega_{1}$. Then $L_{2}:=\psi\left(L_{1}\right)$ is a projective line and $\omega_{2}:=\omega_{1} \circ \psi^{-1}$ defines a projective scale on $L_{2}$.

There are "special" projective isomorphisms with a nice geometrical interpretation of their action on a given projective line. They make the Connection Lemma an essential tool of our matroid construction below. Given a projective line $L$, we call a projective isomorphism $\psi$ special (for $L$ ) if $L$ contains a fixed point of $\psi$. The following concept characterizes the action of a special isomorphism on the associated line.

Definition 5. Let $L_{1}, L_{2} \subset \mathbb{P}^{2}$ be different projective lines. Then a mapping $\varphi: L_{1} \rightarrow$ $L_{2}$ is called a perspective if a point $Q \notin L_{1} \cup L_{2}$ exists such that $x, \varphi(x), Q$ are collinear for all $x \in L_{1}$, i.e., they lie on one projective line. The point $Q$ is called the center of perspective.

Figure 1 illustrates a perspective in charts. In Fig. 1(a) we have used a chart $\varphi_{v}$ in the general position. Let $P$ denote the intersection point of $L_{1}$ and $L_{2}$. The special case $v \perp Q, v \perp P$ is sketched in Fig. 1(b), and the situation $v \perp Q, v \perp P$ is shown in Fig. 1(c).

Lemma 6. Let $L_{1}, L_{2} \cup \mathbb{P}^{2}$ be different projective lines. Then a mapping $\varphi: L_{1} \rightarrow L_{2}$ is a perspective if and only if it extends to a projective isomorphism which is special for $L_{1}$.

Proof. $\quad(\Rightarrow)$ By assumption there is a chart $\varphi_{v}$ with $v \perp Q$ and $v \perp P$ (see Fig. 1(c)), where $\{P\}=L_{1} \cap L_{2}$. In this chart $\varphi$ obviously extends to a shifting $A_{v} \rightarrow A_{v}$. Chosing an appropriate orthogonal coordinate system of the Euclidean space, we have $A_{v}=\mathbb{R}^{2} \times\{1\}$. Then we can write the above shifting as $(x, 1) \mapsto(x, 1)+w$ with $w \in \mathbb{R}^{2} \times\{0\}$. This shifting can be extended to a linear isomorphism of $\mathbb{R}^{3}$ in the following way: $(x, y) \mapsto(x, y)+y w=(x, 0)+y((0,1)+w)$.

$(\Leftarrow)$ (We do not use this part in this paper. However, the proof is added for completeness.) Let $\psi$ be induced by the linear isomorphism $A: \mathbb{R}^{3} \rightarrow \mathbb{R}^{3}$. Choose a point $x \in l_{P}$ different from the origin, where $l_{P}$ denotes the line in $\mathbb{R}^{3}$ corresponding to the intersection point $P$. By assumption, we can replace $A$ by a scalar multiple (if necessary) such that $A(x)=x$. Let $l_{1}$ denote a line through $x$ in $P_{L_{1}}$ which does not meet the origin. 
(Here $P_{L_{i}}$ denotes the plane in $\mathbb{R}^{3}$ inducing the projective line $L_{i}$.) Put $l_{2}:=A\left(l_{1}\right)$. Obviously we have $P_{L_{i}}=$ aff $\left(0, l_{i}\right)$ where aff stands for the affine hull. Since $x \in l_{1} \cap l_{2}$, the affine space aff $\left(l_{1}, l_{2}\right)$ is a plane. This plane cannot contain the origin; otherwise it would coincide with both $P_{L_{i}}$, which are different by assumption. Hence, aff $\left(l_{1}, l_{2}\right)=A_{v}$ for some $v$. In chart $\varphi_{v}$ we have $l_{i}=\varphi_{v}\left(L_{i}\right)$ and the projective isomorphism $\psi$ coincides on $l_{1}$ with $A:\left.l_{1} \rightarrow l_{2} \cdot A\right|_{A_{v}}$ is an affine linear mapping sending $x \mapsto x$. Consequently, the lines connecting $y \in \varphi_{v}\left(L_{1}\right)=l_{1}$ with $\varphi_{v} \circ \psi \circ \varphi_{v}^{-1}(y)=A(y) \in l_{2}$ are either parallel or intersect in one point. Therefore, the corresponding projective lines do intersect in some point $Q$.

Lemmas 6 and 4 together imply the following corollary.

Corollary 7. Let $\varphi: L_{1} \rightarrow L_{2}$ be a perspective between projective lines in $\mathbb{P}^{2}$. Then $\omega_{1}$ is a projective scale on $L_{1}$ if and only if $\omega_{1} \circ \varphi^{-1}$ is a projective scale on $L_{2}$.

The following lemma states that projective scales on arbitrary projective lines can be transferred into each other by means of projective isomorphisms. (We are still able to choose the action in the second dimension arbitrarily.)

Lemma 8. Let $L_{1} \neq \tilde{L}_{1}$ and $L_{2} \neq \tilde{L}_{2}$ be projective lines such that $0_{i} \in L_{i} \cap \tilde{L}_{i}$. Let $0_{i}, 1_{i}, \infty_{i}$ denote distinct points on $L_{i}$. Then a projective isomorphism $\psi$ exists such that $\psi: 0_{1} \mapsto 0_{2}, 1_{1} \mapsto 1_{2}, \infty_{1} \mapsto \infty_{2}$, and $\tilde{L}_{1} \rightarrow \tilde{L}_{2}$.

Proof. We choose a chart $\varphi_{v}$ with $v \perp \infty_{i}, i=1,2$. In $A_{v}$ we find an affine isomorphism $B$ sending $0_{1} \mapsto 0_{2}, 1_{1} \rightarrow 1_{2}$, and $\tilde{L}_{1} \rightarrow \tilde{L}_{2}$. In appropriate coordinates of $\mathbb{R}^{3}$ (compare the proof of Lemma 6 ) we have $A_{v}=\mathbb{R}^{2} \times\{1\}$ and the affine linear isomorphism is given by $(x, 1) \mapsto(B(x), 1)$. This extends to an affine linear isomorphism of $\mathbb{R}^{3}$ by setting $(x, y) \mapsto(B(x)+(y-1) B(0), y)$. Since the origin is a fixed point, the latter is a linear isomorphism.

It is convenient to define (realizable) rank-three oriented matroids by means of point configurations in the affine plane. In this way we introduce below geometrical models for arithmetical operations.

Lemma 9. Assume a point configuration $P=\left(0,1, x, z, \infty, y,-1, F_{1}, F_{2}, F_{3}\right.$, $C_{1}, C_{2}$ ) with an underlying oriented matroid as shown in Fig. 2, see Remark 2. Let $\omega$ denote the projective scale on $L$ with $\omega(0)=0, \omega(1)=1$, and $\omega(\infty)=\infty$. (This kind of scale is referred to as the standard projective scale on $L$.) Then $\omega(z)=\omega(x)-\omega(y)$ holds.

Proof. We consider $\mathbb{R}^{2}$ as a (specific) chart of $\mathbb{P}^{2}$ and regard the same configuration in another chart. For the new chart we choose the line through $\infty, F_{1}, F_{2}$ as the line at infinity. Then lines meeting on aff $\left(\infty, F_{1}\right)$ will appear parallel and $\omega$ is an affine isomorphism $L \rightarrow \mathbb{R}$. (The latter is true since the affine isomorphism is one possible candidate for a projective scale, and the projective scale with fixed entries $0,1, \infty$ 


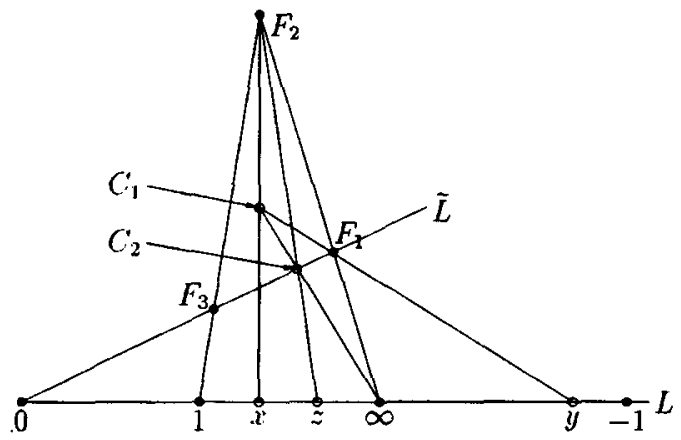

Fig. 2. Operation + .

is unique.) Figure 3 shows the configuration in the new chart. This makes the proof obvious.

Note that the points -1 and $F_{3}$ do not influence the position of the output point $z$ of our configuration. The latter are examples for additional "frame points" which enable us to control the "variable lines." The same transformation as performed in the proof of Lemma 9 (see Fig. 5, below) yields $\omega(z)=-\omega(x)$ (for the standard projective scale on $L$ ). The following lemma implies a constant oriented matroid of operation - for all entries from $(2, \infty)$.

Lemma 10. Consider a configuration which has the following properties in common with that shown in Fig. 4: the collinearities which are marked by lines are present and the points on these lines appear in the described order. Assume that $\omega(x)>2$. Then the underlying oriented matroid is well-determined. In particular, there are the following signed circuits: $\left(\left\{x, C_{1}\right\},\left\{F_{3}, C_{2}\right\}\right)$ and $\left(\left\{z, F_{4}\right\},\left\{F_{3}, C_{2}\right\}\right)$.

Proof. The only two questions not being trivial are the following:

(1) Are $F_{3}$ and $C_{2}$ on the same side of aff $\left(x, C_{1}\right)$ ?

(2) Are $F_{3}$ and $C_{2}$ on the same side of aff $\left(z, F_{4}\right)$ ?

If we keep all points which are marked with the symbol $\bullet$ fixed, then for large values of $\omega(x)$ the asserted signed circuits are present. Let $\omega(x) \downarrow 2$ by means of a homotopy.

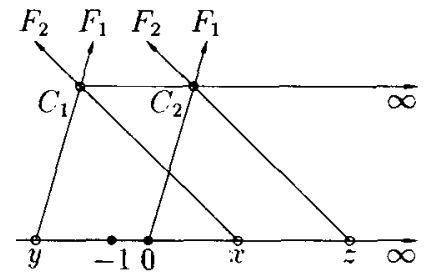

Fig. 3 


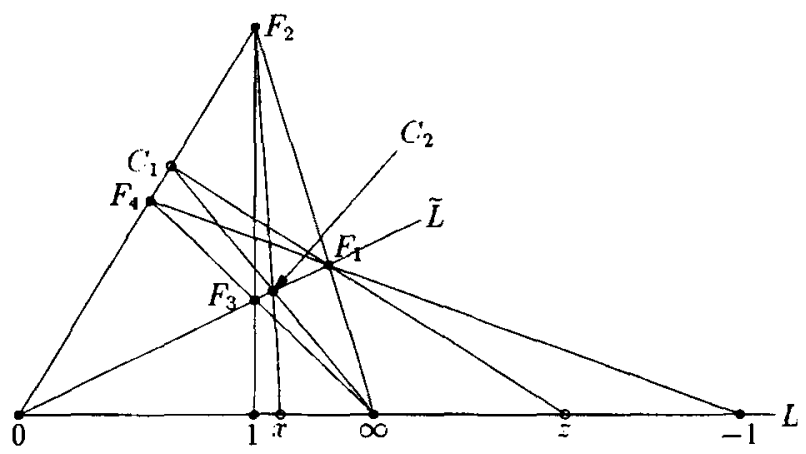

Fig. 4. Operation -.

We are done if we can exclude collinearity of $x, C_{1}, F_{3}$ and $z, F_{4}, C_{2}$ on the whole homotopy. To this end we use a chart of $\mathbb{P}^{2}$ with line aff $\left(\infty, F_{1}\right)$ at infinity, as was done in the proof of Lemma 9. Then $\omega$ is an affine isomorphism. The resulting configuration is shown in Fig. 5. For clearness of argumentation we mark the points -2 and 2 . The lines $l_{1}(x)=a f f\left(x, C_{1}\right)$ and $l_{2}(x)=a f f\left(z, C_{2}\right)$ tend in a parallel and strictly monotonic manner to a line through $F_{3}$ and $F_{4}$, respectively. This completes the proof.

Lemma 11. Assume a point configuration with an underlying oriented matroid as shown in Fig. 6. Let $\omega$ denote the standard projective scale on $L$. Then it holds that $\omega(z)=\omega(x) / \omega(y)$.

Proof. We first introduce a projective scale $\tilde{\omega}$ on $\tilde{L}$, given by $\tilde{\omega}(0)=0, \tilde{\omega}\left(F_{3}\right)=1$, and $\tilde{\omega}\left(F_{1}\right)=\infty$. The point $F_{2}$ induces a perspective between $\omega$ and $\tilde{\omega}$. In view of Corollary 7 it remains to prove that $\tilde{\omega}\left(C_{3}\right)=\tilde{\omega}\left(C_{1}\right) / \omega(y)$. As in the proof of Lemma 9 , we use a new chart of the projective plane to make the assertion obvious. The new chart is chosen such that the line aff $\left(\infty, F_{1}\right)$ is at infinity. The resulting image of the essential points and lines is shown in Fig. 7.

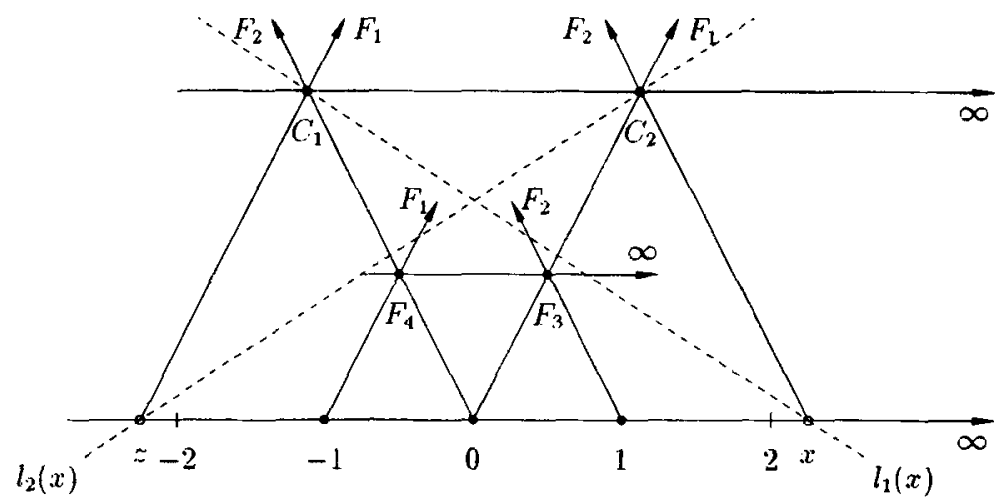

Fig. 5 


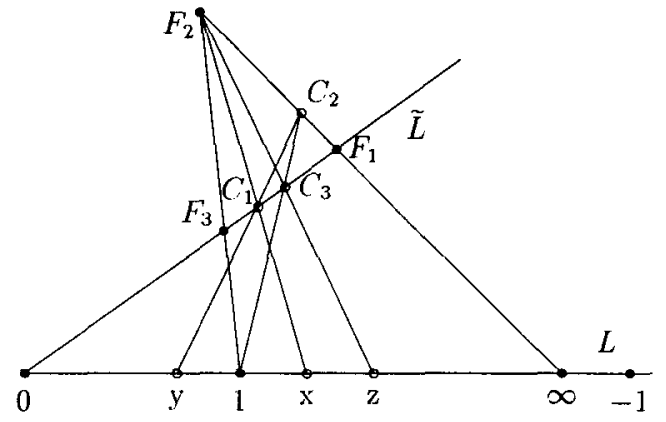

Fig. 6. Operator $x$.

\section{Computations Using Frames}

Until now our geometrical model of addition $(z=x-y)$ requires one input entry from $(1, \infty)$ and one from $(-\infty,-1)$. We have chosen this kind of geometrical model since it guarantees a fixed underlying oriented matroid for all entries it is defined for. This property is the basis for the existence of the mapping $\psi$ in the Universal Partition Theorem. However, the oriented matroid changes in limit situations. For example, in the case $y=-1$ the oriented matroid of the operation + is different from that for input entries $y \in(-\infty,-1)$. Hence, in order to compute arbitrary polynomials, we need an extension of our "catalogue" of elementary computations (by limit situations).

Then we subdivide the points of the configuration into so-called frame points and variable points. Finally, in this section, we fix a computation strategy for $f_{1}, \ldots, f_{m}$. We use a system of storage lines and elementary geometrical computations (such as + and $x$ ), one for each of the intermediate results. We introduce so-called transmissions to guarantee that the input and output entries of an elementary computation coincide with the entries on the corresponding storage lines. Here we apply the Connection Lemma. The system of frames obtained for storage, elementary computation, and transmission builds up our computation frame.

In the following we regard limit situations of the geometrical operations,,$+- \times$. Although the limit situations exhibit a different oriented matroid, the assertions concerning arithmetical operations remain valid.

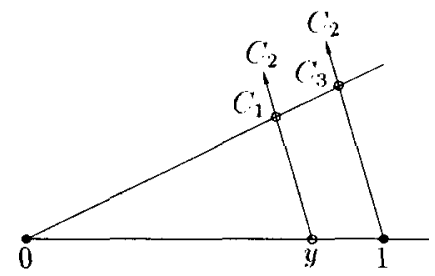

Fig. 7 


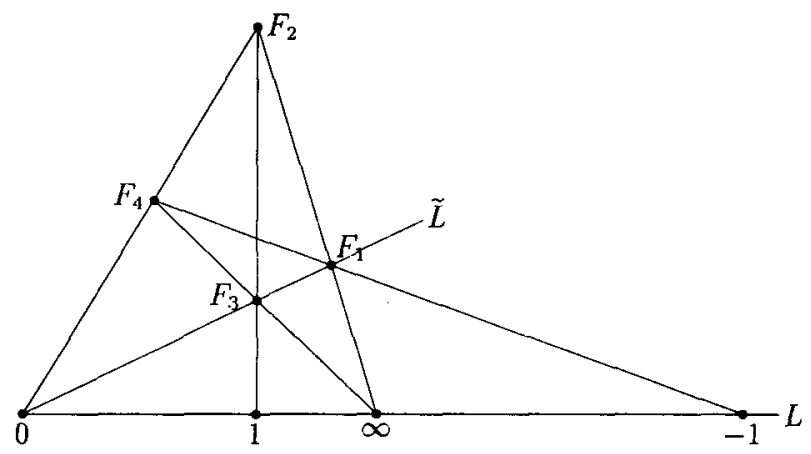

Fig. 8. Operation -1 (limit situation of - ).

With a standard projective scale on $L$ in Fig. 8 it holds that $\omega(-1)=-1$. In Fig. 9 we have $\omega(z)=\omega(x)+1$, and in Fig. 10 we have $\omega(2)=2$. Figure 11 has the limit situation of Fig. 6 for $C_{1} \rightarrow F_{3}$, hence $\omega(z)=1 / \omega(x)$ holds.

Now we have defined the (geometrical) operations $+, \times,-,-1, \uparrow, 2, /$. It is clear that the oriented matroid belonging to the latter configurations does not depend on the particular choice of the input $\omega(x), \omega(y)$ in the corresponding interval $(2, \infty)$ or $(-\infty,-1)$. In all these configurations we have marked so-called frame points with the symbol $\bullet$; the points marked with the symbol $\circ$ are referred to as variable points. The essence of this distinction is the following. Having chosen fixed frame points of an elementary computation (with corresponding submatroid) then, for any choice of admissible input points $x, y$, the other variable points are uniquely determined. Moreover, they depend smoothly on the frame points as well as on the input $\omega(x), \omega(y)$. In case of operation + , for instance, the variable points can be "constructed" by means of intersections of lines in the following order: $C_{1}, C_{2}, z$.

Now we can start to consider the polynomials appearing in our theorem. In general we are not able to use a whole line for storage of the input variables $x_{i}$, since this can

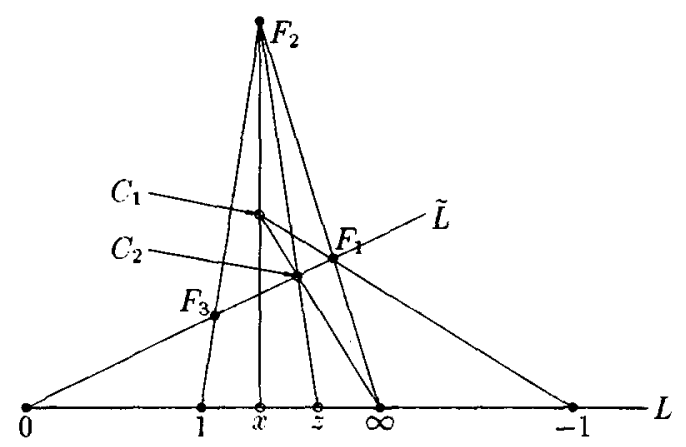

Fig. 9. Operation $\uparrow$ (limit situation of + ). 


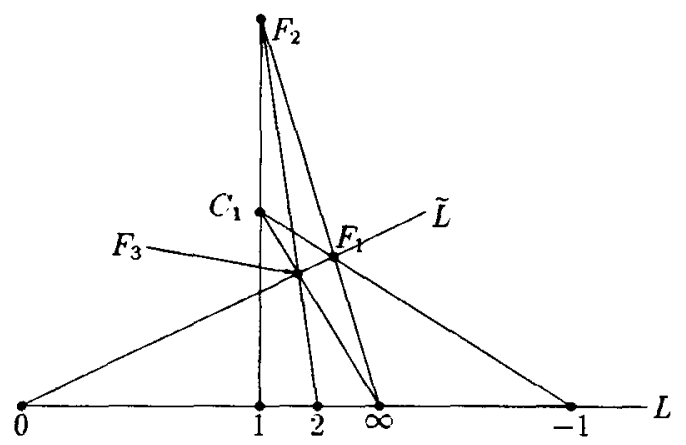

Fig. 10. Operation 2 (limit situation of + and $\uparrow$ ).

yield different oriented matroids for points $x$ from the same elementary semialgebraic set. Therefore, we use an equivalent reformation of this theorem:

Proposition 12. For a proof of the Universal Partition Theorem it suffices to show the theorem which arises if we replace $\mathbb{R}^{n}$ in its formulation by the interval $(2, \infty)^{n}$.

Proof. Let $f_{i}$ be as in the assumption of the Universal Partition Theorem. We apply the new theorem to the polynomails in $u, v$ given by $f_{i}(u-v)$. Then there are an injective mapping $\psi:\{-1,0,1\} \rightarrow \mathcal{M}_{3}(k), \psi: \sigma \mapsto \mathcal{M}_{\sigma}$, a smooth manifold $N_{2}$, and a smooth embedding $\varphi_{2}:(2, \infty)^{2 n} \times N_{2} \rightarrow\left(\mathbb{R}^{2}\right)^{k}$ such that $\mathcal{R}\left(\mathcal{M}_{\sigma}\right)=\varphi_{2}\left(\hat{M}_{\sigma} \times N_{2}\right)$, where

$$
\hat{M}_{\sigma}:=\left\{(u, v) \in(2, \infty)^{n} \times(2, \infty)^{n} \mid \operatorname{sign} f_{i}(u-v)=\sigma_{i}\right\}
$$

It suffices to show the existence of a diffeomorphism $\varphi_{1}:(2, \infty)^{2 n} \rightarrow \mathbb{R}^{n} \times(0, \infty)^{n}$ such that $\left.\varphi_{1}\left(\hat{M}_{\sigma}\right)=M_{\sigma} \times(0, \infty)^{n}\right)$. Then the assertion of the Universal Partition Theorem is established by setting $N:=(0, \infty)^{n} \times N_{2}$ and $\varphi:=\varphi_{2} \circ\left(\varphi_{1}^{-1} \times I d_{N_{2}}\right)$, where $I d_{N_{2}}$ denotes the identical mapping on $N_{2}$.

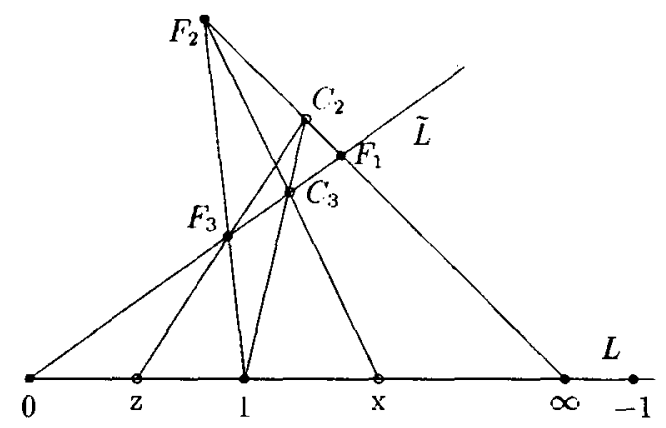

Fig. 11. Operation / (limit situation of $x$ ). 
The diffeomorphism $\varphi_{1}$ can be constructed as the composition of the following two diffeomorphisms $\varphi_{1}=\varphi_{1}^{2} \circ \varphi_{1}^{1}$ :

$$
\varphi_{1}^{1}:\left\{\begin{array}{l}
(2, \infty)^{n} \times(2, \infty)^{n} \rightarrow \tilde{\mathbb{R}}^{2 n}:=\left\{(x, y) \in \mathbb{R}^{n} \times \mathbb{R}^{n}\left|y_{i}>\right| x_{i} \mid\right\}, \\
(u, v) \mapsto(u-v, u+v-4)
\end{array}\right.
$$

and

$$
\varphi_{1}^{2}:\left\{\begin{array}{l}
\tilde{\mathbb{R}}^{2 n} \rightarrow \mathbb{R}^{n} \times(0, \infty)^{n}, \\
(x, y) \mapsto(x, y(y-x)) .
\end{array}\right.
$$

The mapping $\left(\varphi_{1}^{2}\right)^{-1}$ sends $\left\{x_{i}\right\} \times(0, \infty)$ onto $\left\{x_{i}\right\} \times\left\{z_{i} \in \mathbb{R}\left|z_{i}>\right| x_{i} \mid\right\}$, thus it leaves the design variable $x$ of our semialgebraic sets unchanged. In virtue of $\left(\varphi_{1}^{1}\right)^{-1}$, the design variable $x$ appears implicitly (as $u-v$ ) in the definition of $\hat{M}_{\sigma}$.

We prove the new theorem (with $\mathbb{R}^{n}$ replaced by $(2, \infty)^{n}$ ). In the following we find out in which order the elementary computations can be used to compute $f_{1}, \ldots, f_{m} \in \mathbb{Z}[X]$. For this purpose we need some notation (to be used only in Definition 13, below):

$$
\begin{aligned}
\mathbb{N}[X]^{*} & :=\mathbb{N}[X] \backslash\{0\} \quad \text { (nonvanishing polynomials with nonnegative coefficients), } \\
\mathbb{N}[X]_{*} & :=\mathbb{N}[X] \backslash\{0,1\}, \\
\mathbb{N}[X]_{*}^{-1} & :=\left\{1 / f \mid f \in \mathbb{N}[X]_{*}\right\}, \\
\mathbb{Q}(X)_{*} & :=\mathbb{N}[X]^{*} \cup\left(-\mathbb{N}[X]^{*}\right) \cup \mathbb{N}[X]_{*}^{-1} \subset \mathbb{Q}(X) .
\end{aligned}
$$

Definition 13. Let $\mathcal{G}=\left\{g_{1}, g_{2}, \ldots, g_{\alpha}\right\}$ denote a finite sequence in $\mathbb{Q}(X)_{*}$ with pairwise different members. Assume that $\mathcal{E}=\left\{1, X_{1}, \ldots, X_{m}\right\}$ is a subsequence and -1 , 2 are elements of $\mathcal{G}$. An element $g_{i}$ is called a predecessor of $g_{j}$ if $i<j$ holds. The sequence $\mathcal{G}$ is called complete if for any $g \in \mathcal{G} \backslash \mathcal{E}$ a subsequence $\mathcal{P}(g)$ of predecessors and an (arithmetical) operation $\pi(g) \in\{+,-, \times, /\}$ exist such that the following hold:

$\pi(g)=+\Rightarrow \mathcal{P}(g)=\{f, h], \quad f \in \mathbb{N}[X]^{*}, \quad h \in-\mathbb{N}[X]^{*}, \quad g=f-h, \quad$ and $f=1 \Rightarrow h=-1$,

$\pi(g)=-\Rightarrow \mathcal{P}(g)=\{f\}, \quad f \in \mathbb{N}[X]^{*}, \quad$ and $\quad g=-f$,

$\pi(g)=\times \Rightarrow \mathcal{P}(g)=\{f, h], \quad f \in \mathbb{N}[X]_{*}, \quad h \in \mathbb{N}[X]_{*}^{-1}, \quad$ and $\quad g=f / h$,

$\pi(g)=/ \Rightarrow \mathcal{P}(g)=\{f\}, \quad f \in \mathbb{N}[X]_{*}, \quad$ and $\quad g=1 / f$.

We say that a finite sequence $\mathcal{F} \subset \mathbb{Q}(X)_{*}$ is completed by $\mathcal{G}$ if $\mathcal{G}$ is complete and $\mathcal{F}$ is a subsequence of $\mathcal{G}$.

Lemma 14. Any finite sequence $\mathcal{F} \subset \mathbb{N}[X]^{*} \backslash \mathcal{E}$ with pairwise different members can be completed.

Proof. Obviously, $\mathcal{F}$ is subsequence of a sequence $\mathcal{H}=\left\{1, h_{1}, \ldots, h_{\beta}\right\} \subset \mathbb{N}[X]^{*}$ (including $\mathcal{E} \cup\{2\}$ ) with pairwise different members, such that any element of $\mathcal{H} \backslash \mathcal{E}$ is the sum or product of predecessors. By definition, $\left\{1, h_{1},-h_{1}, h_{1}^{-1}, \ldots, h_{\beta},-h_{\beta}, h_{\beta}^{-1}\right\}$ is a complete sequence containing $\mathcal{F}$. 
Consider the polynomials $f_{1}, \ldots, f_{m} \in \mathbb{Z}[X]$ used in our theorem. There are $f_{i}^{+}, f_{i}^{-} \in$ $\mathbb{N}[X]^{*} \backslash \mathcal{E}$ such that $f_{i}=f_{i}^{+}-f_{i}^{-}$. From now on we $f i x$ the family

$$
\mathcal{F}:=\left\{f_{1}^{+}, f_{1}^{-}, \ldots, f_{m}^{+}, f_{m}^{-}\right\}
$$

and a sequence $\mathcal{G}=\left\{g_{1}, \ldots, g_{\alpha}\right\}$ completing it. Also assume that $\mathcal{P}(g)$ and $\pi(g)$ are fixed for $g \in \mathcal{G} \backslash \mathcal{E}$.

Recall that $|\mathcal{G}|=\alpha$. Put $\beta:=\alpha+(\alpha-m-1)$. Let $\xi:\{1, \ldots, \beta\} \rightarrow \mathcal{G} \oplus(\mathcal{G} \backslash \mathcal{E})$, $\xi: i \mapsto g_{i}$ a bijection such that $\xi:\{1, \ldots, \alpha\} \rightarrow \mathcal{G}$. (Here, $\oplus$ denotes the disjoint union.) Definitions 15 and 16 are followed by an interpretation of their meaning.

Definition 15. A configuration of distinct points in $\mathbb{R}^{2}$ is called a basic frame for $\mathcal{G}$ if it consists of the following subconfigurations:

(1) $\left(0^{i}, 1^{i}, \infty^{i}\right)$ with $1^{i} \in\left(0^{i}, \infty^{i}\right)$ for $i=1, \ldots, \beta$. Here $\left(0^{i}, \infty^{i}\right)$ denotes the open line segment. (The line $L^{i}:=\operatorname{aff}\left(0^{i}, \infty^{i}\right)$ is called a storage line for $i=1, \ldots, \alpha$; for $i>\alpha$ it will be extended to an (elementary) computation frame.)

(2) $\left(0_{\{i, j\}}, 1_{j}^{i}, \infty_{j}^{i}, 1_{i}^{j}, \infty_{i}^{j}, Q_{\{i, j\}}\right)$ with $1_{j}^{i} \in\left(0_{\{i, j\}}, \infty_{j}^{i}\right), 1_{i}^{j} \in\left(0_{\{i, j\}}, \infty_{i}^{j}\right)$ and $\left\{Q_{\{i, j\}}\right\}=\operatorname{aff}\left(1_{j}^{i}, 1_{i}^{j}\right) \cap \operatorname{aff}\left(\infty_{j}^{i}, \infty_{i}^{j}\right)$ for all pairs $(i, j)$ satisfying one of the following conditions:

- $i \in\{\alpha+1, \ldots, \beta\}$ and $j \in\{1, \ldots, \alpha\}$ with $g_{j} \in \mathcal{P}\left(g_{i}\right)$ (transmissions for input).

- $i \in\{\alpha+1, \ldots, \beta\}$ and $j \in\{1, \ldots, \alpha\}$ with $g_{i}=g_{j}$ (transmissions for output).

- $i, j \in\{1, \ldots, \alpha\}$ with $g_{i} \in\{-1,2\}$ and $g_{j} \in\left\{X_{1}, \ldots, X_{n}\right\}$ (establishing $\omega(-1)=-1$ and $\omega(2)=2)$.

- $i, j \in\{1, \ldots, \alpha\}$ with $g_{i}=f_{k}^{+}$and $g_{j}=f_{k}^{-}$for some $k \in\{1, \ldots, m\}$ comparison of the final results).

(3) $\left(Q_{j}^{i}\right)$ with $\left\{Q_{j}^{i}\right\}=\operatorname{aff}\left(0^{i}, 0_{\{i, j\}}\right) \cap \operatorname{aff}\left(1^{i}, 1_{j}^{i}\right) \cap$ aff $\left(\infty^{i}, \infty_{j}^{i}\right)$ for all $(i, j)$ and $(j, i)$ from (2). (The lines $L_{j}^{i}$ and $L_{i}^{j}$ are called transmission lines, where $L_{j}^{i}:=$ aff $\left(0_{\{i, j\}}, \infty_{j}^{i}\right)$, etc. $)$

Definition 16. Let a basic frame for $\mathcal{G}$ be given. Assume that the storage lines $L^{i}$ and the transmission lines $L_{j}^{i}$ contain additional points:

- $-1^{i}$ with $\infty^{i} \in\left(1^{i},-1^{i}\right)$ and $\omega^{i}\left(-1^{i}\right)=-1$ for all $L^{i}$.

- $-1_{j}^{i}$ with $\infty_{j}^{i} \in\left(1_{j}^{i},-1_{j}^{i}\right)$ and $\omega_{j}^{i}\left(-1_{j}^{i}\right)=-1$ for all $L_{j}^{i}$.

- $2^{i} \in L^{i}$ with $\omega^{i}\left(2^{i}\right)=2$ for all $i \in\{1, \ldots, \alpha\}$ with $g_{i} \in\left\{X_{1}, \ldots, X_{n}\right\}$.

- $2_{j}^{i} \in L_{j}^{i}$ with $\omega_{j}^{i}\left(2_{j}^{i}\right)=2$ for all $i, j \in\{1, \ldots, \alpha\}$ with $\left\{g_{i}, g_{j}\right\}=\left\{2, X_{k}\right\}$ for some $k$.

Assume in addition that for $i \in\{\alpha+1, \ldots, \beta\}$ the subconfiguration $\left(0^{i}, 1^{i}, \infty^{i}\right)$ extends to a computation frame for the following geometrical operation:

$$
\begin{array}{cl}
-1 & \text { if } \pi\left(g_{i}\right)=- \text { and } \mathcal{P}\left(g_{i}\right)=\{1\}, \\
- & \text { if } \pi\left(g_{i}\right)=- \text { and } \mathcal{P}\left(g_{i}\right) \neq\{1\}
\end{array}
$$




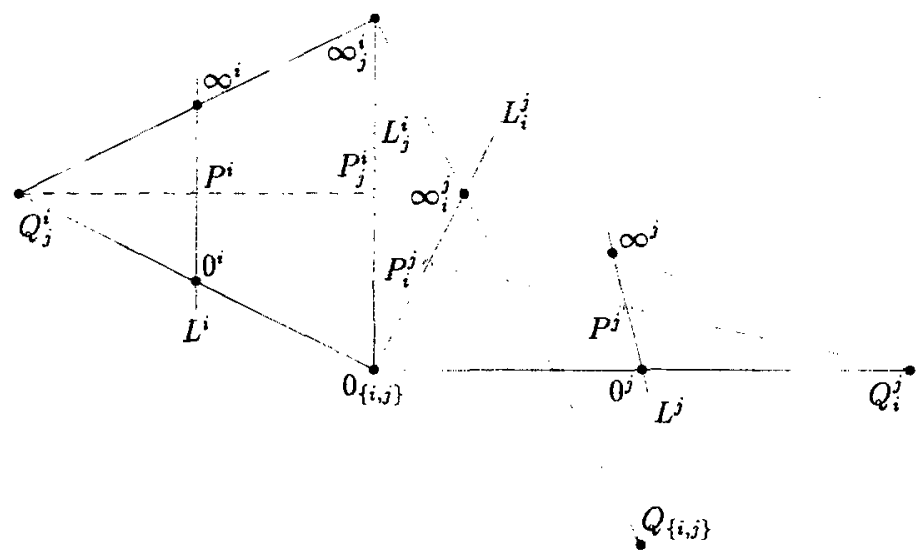

Fig. 12

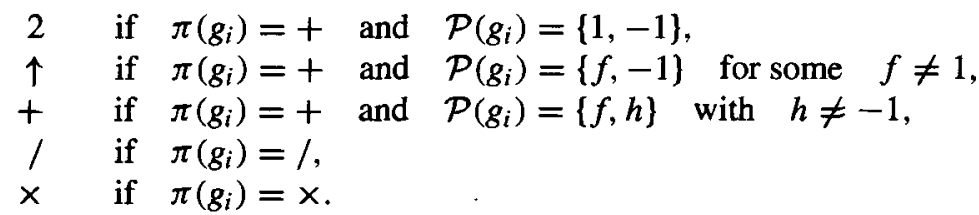

Then the extended configuration is called a $\mathcal{G}$-computation frame.

Now we consider the $\mathcal{G}$-computation frame. We first discuss the basic frame. The basic frame involves lines $L^{i}$ being equipped with standard projective scales. For the pairs $(i, j)$ as explained in (2), we have so-called transmissions between $L^{i}$ and $L^{j}$. A transmission is a chain

$$
L^{i} \rightarrow L_{j}^{i} \rightarrow L_{i}^{j} \rightarrow L^{j}
$$

of perspectives using the center of perspectives $Q_{j}^{i}, Q_{\{i, j\}}$, and $Q_{i}^{j}$. The transmission works as follows. Given a point $P^{i} \in L^{i}$, the first perspective defines the point $P_{j}^{i} \in L_{j}^{i}$ with the same $\omega$-entry. The next step defines $P_{i}^{j}$, and finally we have $P^{j} \in L^{j}$ with $\omega^{j}\left(P^{j}\right)=\omega^{i}\left(P^{i}\right)$, see also Fig. 12 .

The point $-1^{i}$ with $i \leq \alpha$ and $g_{i}=1$ is defined in a twofold way. First, it is defined in Definition 1 by $\omega\left(-1^{i}\right)=-1$ and, second, by means of the computation -1 . However, both definitions coincide. The analogue is true for $2^{i}$ with $i \leq \alpha$ and $g_{i}=2$. By virtue of the Connection Lemma the assumptions concerning entries on standard projective scales $\omega^{i}$ and $\omega_{j}^{i}$ can be replaced by equivalent assumptions using collinearities with center of perspectives $Q_{j}^{i}$ and $Q_{\{i, j\}}$.

\section{How Can a $\mathcal{G}$-Computation Frame Be "Used"?}

Our construction starts with a computation frame $F$ and an input vector $x \in(2, \infty)^{n}$. For $i \leq \alpha$ with $g_{i}=X_{k}(k \in\{1, \ldots, n\})$ we choose $P^{i} \in L^{i}$ such that $\omega^{i}\left(P^{i}\right)=X_{k}(x)=$ 


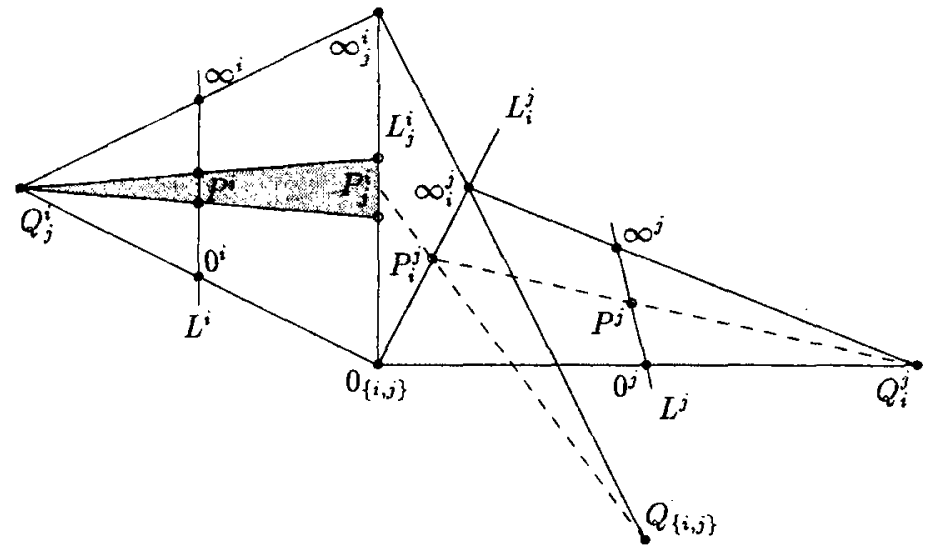

Fig. 13

$x_{k}$. That means that $P^{i}$ represents the value of the input variable $X_{k}$ on its storage line $L_{i}$. The position of the storage points $P^{i}$ with $g_{i} \notin\left\{X_{1}, \ldots, X_{n}\right\}$ is defined inductively. This is done such that the relation $\omega^{i}\left(P^{i}\right)=g_{i}(x)$ is satisfied: Assume that we have already derived $P^{j}$ for all $j<i$. Then the point $P^{i}$ is defined in the following way. First we use the transmissions belonging to the pairs $(i, j)$, with $j>\alpha$ and $g_{j} \in \mathcal{P}\left(g_{i}\right)$, to carry over the input of the operation $\pi\left(g_{i}\right)$ from the storage lines to the corresponding computation frame. Then the frame for the elementary computation is filled in with variable points in the well-defined way yielding an output point. Finally, the $\omega$-entry of the latter is carried over to the storage line $L^{i}$.

The procedure of filling in the computation frame is completed by the use of transmissions belonging to $\left(f_{i}^{+}, f_{i}^{-}\right)$to compare the final results. Here $P_{j}^{i}$ and $P_{i}^{j}$ are defined such that $\omega_{j}^{i}\left(P_{j}^{i}\right)=\omega^{i}\left(P^{i}\right)$ and $\omega_{i}^{j}\left(P_{i}^{j}\right)=\omega^{j}\left(P^{j}\right)$. In contrast to the transmissions used above, the values $\omega^{i}\left(P^{i}\right)$ and $\omega^{j}\left(P^{j}\right)$ do not need to coincide. The latter transmissions are in fact used to compare the function values $f_{i}^{+}(x)$ and $f_{i}^{-}(x)$, see Fig. 13.

The procedure described for "filling in" the computation frame gives rise to the mapping $\varphi:(2, \infty)^{n} \times N \hookrightarrow\left(\mathbb{R}^{2}\right)^{k}$ used in our (new) theorem. Here $N$ stands for a smooth manifold of computation frame. Let $\mathcal{X}=\{1, \ldots, k\}$ denote the index set for the points of a $\mathcal{G}$-computation frame such that the indices $1, \ldots, l$, correspond to the frame points, the indices $l+1, \ldots, l+n$ to the storage points belonging to the input variables $X_{1}, \ldots, X_{n}$, and the remaining indices appear in the (well-defined) order of the construction of the associated points by the filling-in procedure. Given $x \in(2, \infty)^{n}$ and a frame $F \in N \subset \mathbb{R}^{l}$, let $\varphi_{l}:(2, \infty)^{n} \times N \hookrightarrow\left(\mathbb{R}^{2}\right)^{l}$ be defined as the projection mapping onto $N$. Given $\varphi_{i}:(2, \infty)^{n} \times N \hookrightarrow\left(\mathbb{R}^{2}\right)^{i}$ for some index $i \in\{l, \ldots, k-1\}$, we put $\varphi_{i+1}(x, F):=\left(\varphi_{i}(x, F), P^{i+1}\right)$, where $P^{i+1}$ is defined by the filling-in procedure from its predecessors. By induction this implies that $\varphi:=\varphi_{k}$ is a smooth mapping. Since $\varphi_{l+n}$ is an embedding, $\varphi$ is too.

Mapping $\varphi$ is referred to as the "filling in" mapping. It remains to analyze the underlying oriented matroid. 


\section{The Regular Computation Frame}

In Section 4 we have seen how a $\mathcal{G}$-computation frame $F$ for input $x \in(2, \infty)^{n}$ generates a $\mathcal{G}$-computation $\varphi(x, F)$. However, the underlying oriented matroid $\mathcal{M} \circ \varphi(x, F) \in$ $\mathcal{M}_{3}(k)$ was left unclear. In this section we restrict our attention to the subspace $N$ of those $\mathcal{G}$-computation frames which realize a certain oriented matroid $\mathcal{N}$. The oriented matroid $\mathcal{N}$ to be defined (in Section 6 ) will be such that:

(1) $\mathcal{M} \circ \varphi(x, F)$ only depends on sign $f(x)$.

(2) The latter dependence is injective.

(3) $\varphi\left(M_{\sigma} \times N\right)$ coincides with the realization space of the corresponding oriented matroid $\mathcal{R}\left(\mathcal{M}_{\sigma}\right)$ (recall relation (*) in our theorem).

Assuming the existence of such $N \neq \emptyset$, the assertion of our theorem follows immediately: $\psi$ is well-defined by (1), its injectivity is implied by (2), and (3) yields the fundamental relation $(*)$.

The easiest part to prove is (2). Whatever $N$ is, there is a transmission between the storage lines corresponding to $f_{i}^{+}$and $f_{i}^{-}, i=1, \ldots, m$. (Figure 13, above, shows such a transmission.) It is obvious that the oriented matroid of this subconfiguration must be different for inputs from different elementary semialgebraic sets.

In this section we present a sufficient condition for (1) and (3). Therefore we introduce the concept of a regular computation frame.

We first consider a $\mathcal{G}$-computation frame. For proof of existence see Section 6 . This frame decomposes into subframes in a natural way:

For a storage line $L^{i}$ we have the subframe consisting of $\left(0^{i}, 1^{i}, \infty^{i},-1^{i}\right)$.

For an elementary computation $\pi\left(g_{i}\right)$ there is $\left(0^{i}, 1^{i}, \infty^{i},-1^{i}, F_{1}^{i}, F_{2}^{i}, F_{3}^{i},\left(F_{4}^{i}\right)\right)$.

For a transmission we have $\left(0_{i, j}, 1_{j}^{i}, \infty_{j}^{i},-1_{j}^{i}, 1_{i}^{j}, \infty_{i}^{j},-1_{i}^{j}\right)$.

The center of perspective forms such a subframe.

For the latter subframes we define so-called clusters as the convex hull of the points involved. For a storage line $L$ (this time we forget about indices) we define a cluster $\mathcal{L}:=[0,-1]=\operatorname{conv}\{0,-1\}$.

The frames for the elementary computations belonging to the operations $+, \uparrow, 2, \times$, and / coincide (see Fig. 14). For these frames we define the following clusters: $\mathcal{L}:=$ $[0,-1]$ and $\mathcal{F}:=\operatorname{con} v\left\{F_{1}, F_{2}, F_{3}\right\}$. The following set plays a crucial role in the analysis of the underlying oriented matroid of a computation. It is called the forbidden region of the corresponding operation. The forbidden region is defined by setting aff $(\mathcal{L}, \mathcal{F}):=$ $\bigcup_{P_{1} \in \mathcal{L} . P_{2} \in \mathcal{F}} a f f\left(P_{1}, P_{2}\right)$. It is illustrated in Fig. 14. A moment of reflection shows that for each of the above computations any line connecting two configuration points, not both belonging to $\mathcal{L}$, meets both $\mathcal{L}$ and $\mathcal{F}$, i.e., it is contained in the forbidden region. Later we will see that control of the forbidden regions can be used to control the oriented matroid of a $\mathcal{G}$-computation. It is an important feature of aff $(\mathcal{L}, \mathcal{F})$ that it does not contain any line parallel to $L$ (this fact establishes our control of the forbidden regions). 


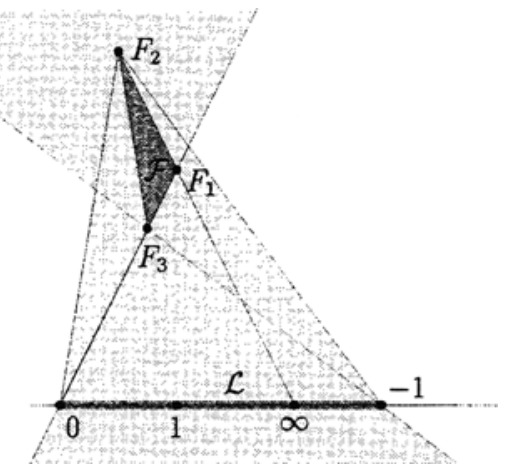

Fig. 14

The computation frames for the operations - and -1 coincide too. Moreover, the subframe consisting of all points except $F_{4}$ is just the frame for + , etc. Here we have the clusters $\mathcal{L}:=[0,-1]$ and $\mathcal{F}:=\operatorname{conv}\left\{F_{1}, F_{2}, F_{3}, F_{4}\right\}$.

In the following we prove that the forbidden region for - and -1, aff $(\mathcal{L}, \mathcal{F})$, coincides with the forbidden region defined for the other operations, i.e., aff $(\mathcal{L}, \mathcal{F})=$ $\operatorname{aff}\left(\mathcal{L}, \operatorname{conv}\left\{F_{1}, F_{2}, F_{3}\right\}\right)$. To this end we have to show that the point $P(\lambda):=\lambda F+$ $(1-\lambda) P$ belongs to aff $\left(\mathcal{L}, \operatorname{conv}\left\{F_{1}, F_{2}, F_{3}\right\}\right)$ for all choices of $F \in \operatorname{conv}\left(F_{2}, F_{3}, F_{4}\right)$, $P \in \mathcal{L}$, and $\lambda \in \mathbb{R}$. For $\lambda \in[0,1]$ this follows from $F_{4} \in \operatorname{aff}\left(\mathcal{L}, \operatorname{con} v\left\{F_{1}, F_{2}, F_{3}\right\}\right)$. For $\lambda \notin[0,1]$ let $L_{2}$ be a line separating $\mathcal{L}$ from $\operatorname{conv}\left\{F_{1}, F_{2}, F_{3}\right\}$, i.e., the above sets lie in the different open half-spaces generated by $L_{2}$. We now regard our affine configuration as the image of a projective configuration under a chart, say $\varphi_{v_{1}}$. We take a new chart $\varphi_{v_{2}}$ such that $L_{2}$ appears as the line at infinity. In the image of the new chart $\lambda \notin[0,1]$ the point $P(\lambda)$ lies in the open line segment $(F, P)$ (recall that $P(\lambda)$ was defined explicitly in chart $\left.\varphi_{v_{1}}\right)$. Moreover, in the new chart we also have $F_{4} \in \operatorname{aff}\left(\mathcal{L}, \operatorname{conv}\left\{F_{1}, F_{2}, F_{3}\right\}\right)$. This again implies $P(\lambda) \in \operatorname{aff}\left(\mathcal{L}\right.$, conv $\left.\left\{F_{1}, F_{2}, F_{3}\right\}\right)$ and we are done. In addition, we see that the forbidden region also contains all connecting lines except $L$.

For a transmission we define the following clusters: $\mathcal{C}_{\{i, j\}}:=\left\{0_{\{i, j\}}\right\}, \mathcal{C}_{j}^{i}:=\left[1_{j}^{i},-1_{j}^{i}\right]$, and $\mathcal{C}_{i}^{j}:=\left[1_{i}^{j},-1_{i}^{j}\right]$. Here we have to control the following forbidden region aff $\left(\mathcal{C}_{j}^{i}, \mathcal{C}_{i}^{j}\right)$, as sketched in Fig. 15. For ease of formulation we also introduce so-called superclusters $\mathcal{S}$ as follows. For a storage line let $\mathcal{S}:=\mathcal{L}$. For an elementary computation put $\mathcal{S}:=$ $\operatorname{conv}(\mathcal{L}, \mathcal{F})$. For a transmission let $\mathcal{S}:=\operatorname{conv}\left(\mathcal{C}_{\{i, j\}}, \mathcal{C}_{j}^{i}, \mathcal{C}_{i}^{j}\right)$. The center of perspectives $\left\{Q_{j}^{i}\right\}$ and $\left\{Q_{\{i, j\}}\right\}$ form clusters and superclusters.

Note that all clusters and superclusters are convex hulls of frame points! This is intrinsically used in the proof of Proposition 18, below.

Definition 17. A $\mathcal{G}$-computation frame is called regular if the following conditions are satisfied:

- The clusters are pairwise disjoint.

- For $i=1, \ldots, \beta$ the supercluster $\mathcal{S}^{i}$ is the only one meeting $L^{i}$. 


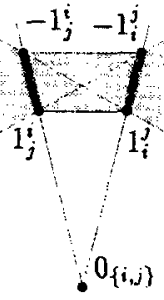

Fig. 15

- For any pair of different clusters $\left(\mathcal{C}_{a}, \mathcal{C}_{b}\right)$ not contained in supercluster $\mathcal{S}_{c}$ there is a cluster $\mathcal{C}_{c} \subset \mathcal{S}_{c}$ such that $\operatorname{aff}\left(\mathcal{C}_{a}, \mathcal{C}_{b}\right) \cap \mathcal{S}_{c}=\operatorname{aff}\left(\mathcal{C}_{a}, \mathcal{C}_{b}\right) \cap \mathcal{C}_{c}$. If the latter intersection is nonempty, then two of the clusters are line segments and the other one is the corresponding center of perspective.

By virtue of Proposition 18 it suffices to show the existence of a regular computation frame $F$ in order to prove our theorem.

Proposition 18. Let $F$ denote a regular $\mathcal{G}$-computation frame and put $\mathcal{N}:=\mathcal{M}(F)$. Then $N:=\mathcal{R}(\mathcal{N})$ is a smooth manifold and the "filling in" mapping $\varphi$ satisfies relations (1)-(3).

\section{Proof.}

Step 1 ( $N$ is a smooth manifold). By definition it is a property of the underlying oriented matroid whether or not a configuration is a $\mathcal{G}$-computation frame. A moment of reflection shows that the set $\mathcal{R}(\mathcal{N})$ is open in the locus of (all) $\mathcal{G}$-computation frames. In order to obtain a $\mathcal{G}$-computation frame, the points $0^{i}, \infty^{i}, F_{1}^{i}, 0_{\{i, j\}}$, and $\infty_{j}^{i}$ can be chosen in general position (with respect to collinearity). Their choice uniquely defines the center of perspectives of form $Q_{j}^{i}$. Then, for $i=1, \ldots, \beta$, the point $1^{i}$ can be chosen in general position on the open line segment $\left(0^{i}, \infty^{i}\right)$. For each $i$ this determines the position of $1_{j}^{i}$ for those $j$ for which the point $Q_{j}^{i}$ exists. Now the pair $\left(1_{j}^{i}, 1_{j}^{j}\right)$ is fixed and the center of perspective $Q_{\{i, j\}}$ is well-defined. Finally, the choice of $F_{2}^{i}$ in general position under the restriction $F_{1}^{i} \in\left(F_{2}^{i}, \infty^{i}\right)$ defines the position of the remaining points of a $\mathcal{G}$-computation frame. Locally, all points can be chosen in smooth dependence on the data that have been fixed previously and appropriate real parameters. Consequently, the realization space $\mathcal{R}(\mathcal{N})$ of a regular computation frame constitutes a smooth manifold.

Step 2 (we show the relation (1)). In order to organize control of the oriented matroid of a $\mathcal{G}$-computation we consider (small) parts of it which are already controlled by its definition. They are called units and defined in the following way. The subframes defined above for the storage lines (elementary computations) are called storage units (computation units). For each connection (between some $L^{i}$ and $L^{j}$ ) we define three units of perspective, consisting of the points from $L^{i} \cup L_{j}^{i} \cup\left\{Q_{j}^{i}\right\}, L^{j} \cup L_{i}^{j} \cup\left\{Q_{i}^{j}\right\}$, 
and $L_{j}^{i} \cup L_{i}^{j} \cup\left\{Q_{\{i, j\}}\right\}$, respectively. Independently from the choice of $x \in(2, \infty)^{n}$, the value $g(x)$ lies in either $(2, \infty)$ or $(-\infty,-1)$ for arbitrary $g \in \mathcal{G} \backslash\{1,-1,2\}$. Hence, the oriented matroid of a filled-in unit (compare the definition of $\varphi$ ) only depends on sign $f_{i}(x), i=1, \ldots, m$. Note that the units "cover" the whole frame, however, they do not decompose it, in contrast to the subframe above. Generally speaking, the units represent the "tasks" of the parts of our computation frame.

Note that a $\mathcal{G}$-computation using a regular computation frame never exhibits a signed circuit of length 1 or 2 . (Length 1 is already excluded by definition of an oriented matroid. Length 2 cannot appear since all points of such a computation are distinct.) In order to control the underlying oriented matroid of a $\mathcal{G}$-computation we introduce the so-called $k$-test, where $k \in\{3,4\}$. A $k$-test is a subconfiguration of cardinality $k$ of the $\mathcal{G}$-computation which is not contained in one unit, and which does not exhibit a signed circuit of length smaller than $k$. For assertion (1) it suffices to show that the oriented matroid of a 3-test and of a 4-test only depends on $\mathcal{N}$ and sign $f(x)$.

It turns out that a 3-test never exhibits a signed circuit. This can be seen as follows. By definition there is no 3-test contained in one single cluster. If a 3-test would meet precisely two clusters, then that one of them containing two points must be some $\mathcal{F}$. (The other clusters are only one-dimensional: distinguish between $L^{i}$ and $L_{j}^{i}$ !) The other cluster is $\mathcal{L}^{i}$, since $\mathcal{L}^{i}$ is met by any line connecting two configuration points from $\mathcal{F}^{i}$ and there is no other cluster met by aff $\left(\mathcal{L}^{i}, \mathcal{F}^{i}\right)$ (by regularity). Hence, the 3 -test is contained in one unit, which was excluded by its definition. Consequently, any 3-test has points from pairwise different clusters. If it would exhibit a circuit, then it was contained in a perspective unit (by regularity), a contradiction.

Now, let be $P=\left(P_{1}, P_{2}, P_{3}, P_{4}\right)$ be a 4-test and let $\mathcal{C}_{1}, \ldots, \mathcal{C}_{4}$ be the corresponding clusters. Since there is no circuit of length $1,2,3$, the set of signed circuits $\mathcal{C}(P)$ consists of precisely one signed circuit (of length 4 ) and its negative. If for all "permutations" $\{a, b, c, d\}=\{1,2,3,4\}$ it is clear whether or not aff $\left(P_{a}, P_{b}\right)$ separates $P_{c}$ and $P_{d}$, then $\mathcal{C}(P)$ is well-determined. In fact, there is a permutation such that aff $\left(P_{a}, P_{b}\right)$ separates $P_{c}$ and $P_{d}$, i.e., $P_{c}$ and $P_{d}$ lie on different sides of aff $\left(P_{a}, P_{b}\right)$. If $P_{a}$ and $P_{b}$ are separated by aff $\left(P_{c}, P_{d}\right)$, then $\left\{P_{a}, P_{b}\right\}\left\{P_{c}, P_{d}\right\}$ is a signed circuit (identify the configuration points with elements of the basic set of the underlying oriented matroid). If otherwise aff $\left(P_{a}, P_{c}\right)$ separates $P_{b}$ and $P_{d}$, then we have $\left\{P_{a}\right\}\left\{P_{b}, P_{c}, P_{d}\right\}$ and in the remaining case $\left\{P_{b}\right\}\left\{P_{a}, P_{c}, P_{d}\right\}$.

In summary, we have to prove that it only depends on $\mathcal{N}$ and sign $f(x)$ whether or not $\operatorname{aff}\left(P_{1}, P_{2}\right)$ separates $P_{3}$ and $P_{4}$.

By definition of a 4-test the points $P_{1}, \ldots, P_{4}$ do not belong to the same supercluster. If aff $\left(P_{1}, P_{2}\right)$ is one of the lines $L^{i}$ or $L_{j}^{i}$, then aff $\left(P_{1}, P_{2}\right)$ cannot intersect $\mathcal{C}_{3}$ or $\mathcal{C}_{4}$. (If $\mathcal{C}_{3}$ would be intersected, then $\mathcal{C}_{3} \cap L^{i}$ (or $L_{j}^{i}$ ) is nonempty, hence $\mathcal{C}_{3} \subset L_{i}$ (or $L_{j}^{i}$ ), by regularity. This would imply that $P_{1}, P_{2}, P_{3}$ are collinear which was exluded in the definition of a 4-test.) Now, it is clear from that oriented matroid $\mathcal{N}$ of the frame whether $\mathcal{C}_{3}$ and $\mathcal{C}_{4}$ are in the same or in different open half-spaces generated by $L_{i}\left(L_{j}^{i}\right)$. Hence, in that case the oriented matroid $\mathcal{N}$ decides whether or not $L^{i}$ (or $L_{j}^{i}$ ) separates $P_{3} \in \mathcal{C}_{3}$ and $P_{4} \in \mathcal{C}_{4}$.

In the following we assume that $\operatorname{aff}\left(P_{1}, P_{2}\right)$ is different from the lines $L^{i}$ and $L_{j}^{i}$. In the case $\mathcal{C}_{1}=\mathcal{C}_{2}$, cluster $\mathcal{C}_{1}$ must be a two-dimensional one, i.e., some $\mathcal{F}^{i}$. This implies 
that $\operatorname{aff}\left(P 1, P_{2}\right) \cap \mathcal{L}^{i} \neq \emptyset$. In this case put $\tilde{\mathcal{C}}_{1}:=\mathcal{C}_{1}=\mathcal{F}^{i}$ and $\tilde{\mathcal{C}}_{2}:=\mathcal{L}^{i}$. In the case $\mathcal{C}_{1} \neq \mathcal{C}_{2}$ put $\tilde{\mathcal{C}}_{1}:=\mathcal{C}_{1}$ and $\tilde{\mathcal{C}}_{2}:=\mathcal{C}_{2}$.

By regularity of the $\mathcal{G}$-computation frame it only depends on the choice of $P_{i}$ (in the basic set of the underlying oriented matroid, not on the geometrical position!) which of the following cases occurs.

Case 1: aff $\left(\tilde{\mathcal{C}_{1}}, \tilde{\mathcal{C}}_{2}\right) \cap\left(\mathcal{C}_{3} \cup \mathcal{C}_{4}\right)=\emptyset$. Then $\mathcal{C}(P)$ is well-determined by $\mathcal{N}$.

Case 2: aff $\left(\tilde{\mathcal{C}}_{1}, \tilde{\mathcal{C}}_{2}\right)$ meets exactly one of $\mathcal{C}_{3}$ and $\mathcal{C}_{4}$, say $\mathcal{C}_{3}$. Then $P_{1}, P_{2}, P_{3}$ belong to the same unit. It is well-determined by the choice of $P_{1}, P_{2}$ (in the basic set of the underlying oriented matroid) whether or not the point 0 (or $\infty$ ) of the supercluster corresponding to $\tilde{\mathcal{C}}_{1}$ is on aff $\left(P_{1}, P_{2}\right)$. The line aff $\left(P_{1}, P_{2}\right)$ cannot contain both of them since it differs from $L^{i}, L_{j}^{i}$. Choose one of the points $0, \infty$ not lying on aff $\left(P_{1}, P_{2}\right)$ as the orientation point. The oriented matroid of the unit is well-defined by $\mathcal{N}$ and sign $f(x)$. Hence, it is clear whether or not the orientation point and $P_{3}$ are on the same side of aff $\left(P_{1}, P_{2}\right)$. We deduce that it suffices to decide whether or not aff $\left(P_{1}, P_{2}\right)$ separates $\mathcal{C}_{4}$ and the orientation point. Since aff $\left(\tilde{\mathcal{C}}_{1}, \tilde{\mathcal{C}}_{2}\right)$ does not meet $\mathcal{C}_{4}$, these are frame points $\tilde{P}_{1}, \tilde{P}_{2}$ from $\tilde{\mathcal{C}}_{1}$ and $\tilde{\mathcal{C}}_{2}$, respectively, such that the orientation point is separated from $\mathcal{C}_{4}$ by aff $\left(P_{1}, P_{2}\right)$ if and only if it is separated by aff $\left(\tilde{P}_{1}, \tilde{P}_{2}\right)$. The latter is decided by $\mathcal{N}$.

Case 3: aff $\left(\tilde{\mathcal{C}_{1}}, \tilde{\mathcal{C}_{2}}\right)$ meets both $\mathcal{C}_{3}$ and $\mathcal{C}_{4}$. Then all points belong to the same unit, which is not possible by definition of a 4-test.

Step 3 (proof of relation (3)). Let $\sigma \in\{-1,0,1\}^{m}$. By relation (1), the matroid $\mathcal{M}_{\sigma}$ is well-defined and we have $\varphi\left(M_{\sigma} \times N\right) \subset \mathcal{R}\left(\mathcal{M}_{\sigma}\right)$. We show that the latter sets coincide. To this end assume a configuration with underlying matroid $\mathcal{M}_{\sigma}$. Then the subconfiguration corresponding to the frame is from $\mathcal{R}(\mathcal{N})$. Consequently, this is a (regular) $\mathcal{G}$-computation frame. (A regular computation frame is defined by properties of the underlying oriented matroid.) The points $P^{X_{i}}$ must be in $\left(2^{X_{i}}, \infty^{X_{i}}\right)$, by the oriented matroid $\mathcal{M}_{\sigma}$. Moreover, the oriented matroid implies that our configuration is a filled-in $\mathcal{G}$ computation frame, thus a $\mathcal{G}$-computation, with input $x=\left(\omega^{X_{1}}\left(P^{X_{1}}\right), \ldots, \omega^{X_{n}}\left(P^{X_{n}}\right)\right)$. All such computations are in the image of $\varphi$.

\section{The Existence of a Regular Computation Frame}

In this section we construct a regular computation frame. In view of Section 5 this completes the proof of the Universal Partition Theorem. We first construct a family of basic frames. By definition, the basic frame is the part of the computation frame which is responsible for the storage and transportation of the (intermediate) results. It is obtained from the computation frame by deleting the clusters $\mathcal{F}^{i}$, which realize the elementary computations, and the points $-1^{i},-1_{j}^{i}, 2^{i}, 2_{j}^{i}$. Our family of basic frames is such that all lines $L^{i}$ and all points $0^{i}, O_{\{i, j\}}, Q_{j}^{i}$, and $Q_{\{i, j\}}$ are constant. Moreover, the line segments $\left[0^{i}, \infty^{i}\right] \subset L^{i}$ "converge" to the points $0^{i}$ and $\left[0_{\{i, j\}}, \infty_{j}^{i}\right] \subset L_{j}^{i}$ to $0_{\{i, j\}}$. (It is not trivial that this can be done with a fixed center of perspectives.) In a second step we attach the frames used for elementary computations in order to complete the 
computation frame. This can be done in such a way that the superclusters $\mathcal{S}^{i}$ "converge" to $0^{i}$ and the forbidden regions to given lines $\tilde{L}^{i}$. This implies the existence of values for the parameter corresponding to a regular computation frame.

Proposition 19. A regular $\mathcal{G}$-computation frame exists.

Proof. We start our construction with the points $0^{i}, 0_{\{i, j\}}$ (with $i=1, \ldots, \beta$, and $j$ as in Definition 15(2)) such that no triple of these points is collinear. Next, we choose lines $L^{i}$ and $L_{j}^{i}$ such that $0^{i} \in L^{i}\left(0_{\{i, j\}} \in L_{j}^{i}\right)$ is the only one of the latter points met by $L^{i}$ and $L_{j}^{i}$, respectively. This can be done such that the lines $L^{i}, L_{j}^{i}$ with the same index $i$ are parallel, and different lines $L^{i}$ are not parallel.

The existence of continuous one-parametric families of points $A^{i}\left(t^{i}\right) \in L^{i}$ and $A_{j}^{i}\left(t_{j}^{i}\right) \in L_{j}^{i}$ with $t^{i}, t_{j}^{i} \in(0,1)$ such that $\left\|A^{i}\left(t^{i}\right)-0^{i}\right\|=t^{i}$ and $\left\|A_{j}^{i}\left(t_{j}^{i}\right)-0_{\{i, j\}}\right\|=t_{j}^{i}$, where $\|\cdot\|$ denotes the Euclidean norm on $\mathbb{R}^{2}$, is also clear. Later, the points $A^{i}\left(t^{i}\right)$ will be used to define the points $\infty^{i}$ and $1^{i}$ (for different value of the parameter $t^{i}$ ). Analogously, we define the points $\infty_{j}^{i}$ and $1_{j}^{i}$.

We treat $\mathbb{R}^{2}$ as a chart of $\mathbb{P}^{2}$. Hence, for all values of parameters $t^{i}$ and $t_{j}^{i}$ there is precisely one point $Q_{j}^{i}\left(t^{i}, t_{j}^{i}\right)$ on the projective line connecting $0^{i}$ and $0_{\{i, j\}}$ which is collinear with the pair $A^{i}\left(t^{i}\right), A_{j}^{i}\left(t_{j}^{i}\right)$. The position of $Q_{j}^{i}$ (on the projective line corresponding to aff $\left(0^{i}, 0_{\{i, j\}}\right)$ ) depends continuously and strictly monotonically on the ratio $t^{i}: t_{j}^{i}$. This is obvious since $L_{i}$ and $L_{j}^{i}$ are parallel lines. The only point $Q_{j^{\prime}}^{i^{\prime}}$ which depends on the parameter $t_{j}^{i}$ is $Q_{j}^{i}$. Hence, there are ratios of the parameters $t^{i}: t_{j 1}^{i}: \cdots: t_{j r}^{i}, i=1, \ldots, \beta$, such that there are no other collinearities between the points $\left\{0^{i}, 0_{\{i, j\}}, Q_{j}^{i}\right\}_{i, j}$ other than those of the form $\left\{0^{i}, 0_{\{i, j\}}, Q_{j}^{i}\right\}$. We fix such ratios. Now, the parameter $t_{j}^{i}$ is already given by $t^{i}$. In order to emphasize this relation we write $A_{j}^{i}\left(t^{i}\right)$ for $A_{j}^{i}\left(t_{j}^{i}\right)$.

We are still free to choose the ratio $t^{1}: \cdots: t^{\beta}$. It is clear that for a fixed ratio $t^{i}: t^{j}$ all possible lines aff $\left(A_{j}^{i}\left(t^{i}\right), A_{i}^{j}\left(t^{j}\right)\right)$ are parallel. (They are well-defined, since $0_{\{i, j\}}$ is the only common point of $L_{j}^{i}$ and $L_{i}^{j}$ by construction.) The projective lines corresponding to aff $\left(A_{j}^{i}\left(t^{i}\right), A_{i}^{j}\left(t^{j}\right)\right)$ intersect, say in $Q_{\{i, j\}}\left(t^{i} / t^{j}\right)$ on the line at infinity. It is obvious that the position of $Q_{\{i, j\}}$ on the line at infinity depends strictly monotonically and continuously on $t^{i} / t_{j}$. Therefore, there is a ratio $t^{1}: \cdots: t^{\beta}$ for which all small points $Q_{\{i, j\}}\left(t^{i} / t^{j}\right)$ are different (the corresponding lines are not parallel) and for small values of the parameter $t^{1}$ there is only one collinearity of $Q_{\{i, j\}}$ with points from $\left\{0^{i}, 0_{\{i, j\}}, Q_{j}^{i}, A^{i}\left(t^{i}\right), A_{j}^{i}\left(t^{i}\right)\right\}_{i, j}$, namely $A_{j}^{i}\left(t^{i}\right), A_{i}^{j}\left(t^{j}\right), Q_{\{i, j\}}$. (We assume small values of $t^{1}$ in order to control the points $A^{i}$ and $A_{j}^{i}$.) We fix one of these ratios and put $t:=t^{1}$. (From now on we write $A^{i}(t)$ and $A_{j}^{i}(t)$.)

We can choose another chart of $\mathbb{P}^{2}$ such that all points defined so far are in $\mathbb{R}^{2}$. Note that the collinearity of the points $Q_{[i, j]}$ does not fit in our concept of a regular computation frame. Nevertheless, we proceed with an extension of the given configuration to a $\mathcal{G}$ computation frame (being as regular as possible). The collinearities of the points $Q_{\{i, j\}}$ are perturbed at the very end.

Our one-parametric family of configurations, constructed above, defines a two-parametric family of basic frames in the following way: $\infty^{i}\left(t_{\infty}\right):=A^{i}\left(t_{\infty}\right), \infty_{j}^{i}\left(t_{\infty}\right):=$ 


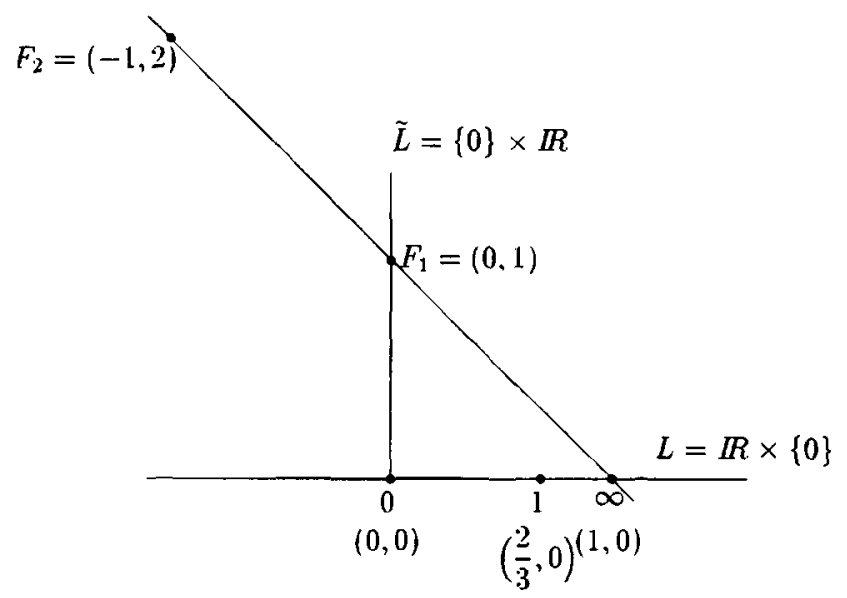

Fig. 16

$A_{j}^{i}\left(t_{\infty}\right)$, and $1^{i}\left(t_{1}\right):=A^{i}\left(t_{1}\right), 1_{j}^{i}\left(t_{1}\right):=A_{j}^{i}\left(t_{1}\right)$, where $0<t_{1}<t_{\infty} \ll 1$. We use the fact that $t^{\infty}$ is sufficiently close to 0 in order to guarantee that $0^{i}, 1^{i}$, and $\infty^{i}$ appear (on $L^{i}$ ) in the right order, etc.

In the next step we attach the frames for the elementary operations,+- , etc. Consider the configuration in $\mathbb{R}^{2}$ shown in Fig. 16. The latter configuration can be extended to any geometrical model for an elementary operation. The corresponding computation frame (with $\omega(-1)=-1$ ) is well-determined by the given points, hence the clusters $\mathcal{L}$ and $\mathcal{F}$ are. It is referred to as the standard frame for the elementary computation. Using a parameter $\xi \in(0,1]$ we obtain a parametric family of elementary computation frames as the image of the standard frame under the linear mapping $\left(x_{1}, x_{2}\right) \mapsto\left(\xi^{2} x_{1}, \xi x_{2}\right)$. The frame for parameter $\xi=1$ coincides with the original one. Obviously, all points of the parametrized frame converge to 0 . The line $L(\xi)=L$ is constant. Since the forbidden region aff $(\mathcal{L}(\xi), \mathcal{F}(\xi)$ ) does not contain any line parallel to $L$ (see Section 5), it follows that

$$
\lim _{\xi \rightarrow 0} \operatorname{aff}(\mathcal{L}(\xi), \mathcal{F}(\xi))=\{\tilde{L}\} .
$$

Here the set $\lim _{\xi \rightarrow 0}$ aff $\left(\mathcal{C}_{1}(\xi), \mathcal{C}_{2}(\xi)\right)$ is defined for parametric families of disjoint sets $\mathcal{C}_{1}(\xi), \mathcal{C}_{2}(\xi) \subset \mathbb{R}^{2}$ as the set of those lines $l$ for which there are sequences $\xi^{i} \rightarrow 0$ and $c_{1}^{i}, c_{2}^{i}$ with $c_{1}^{i} \in C_{1}\left(\xi^{i}\right) c_{2}^{i} \in C_{2}\left(\xi^{i}\right)$ such that $l=\lim _{i \rightarrow \infty}$ aff $\left(c_{1}^{i}, c_{2}^{i}\right)$. The latter is said to hold if $l$ contains an accumulation of aff $\left(c_{1}^{i}, c_{2}^{i}\right)$ and the linear spaces $\mathbb{R}\left(c_{2}^{i}-c_{1}^{i}\right)$ converge in the Grassmannian $G(2,1)$ to the space parallel to $l$.

For $i=\alpha+1, \ldots, \beta$ let $\varphi^{i}: \mathbb{R}^{2} \rightarrow \mathbb{R}^{2}$ denote a projective isomorphism with $\varphi^{i}: 0 \mapsto 0^{i}, 1 \mapsto 1^{i}(1), \infty \mapsto \infty^{i}(1)$, and $\tilde{L} \rightarrow \tilde{L}^{i}$, where the arguments are taken from our standard frame. The existence of $\varphi^{i}$ follows from Lemma 8. For the time being we fix the ratio $t_{1}: t_{\infty}=2: 3$ and put $t=t_{\infty}$. (Then the points $1^{i}(t)$, etc., are well-defined.) Since $\left(A^{i}\right)^{-1}: L^{i} \rightarrow \mathbb{R}$ has been constructed as an affine isomorphism (in the old chart) it is a projective scale on $L^{i}$. This implies $\infty^{i}(t)=\varphi^{i}(t, 0)$ and $1^{i}(t)=\varphi^{i}(2 t / 3,0)$. For small values of the parameter $t$, the oriented matroid of the standard elementary 
computation frame coincides with the oriented matroid of the image. (For this we use an image that is close to $0^{i}$.) It follows the fundamental relation

$$
\lim _{t \rightarrow 0} \operatorname{aff}\left(\mathcal{L}^{i}(t), \mathcal{F}^{i}(t)\right)=\left\{\tilde{L}^{i}\right\}
$$

We also see that $\mathcal{S}^{i}$ collapses to the point $0^{i}$.

Now we put $t_{\infty}:=t$ and $t_{1}:=t-t^{2} / 3$. For $t \in(0,1)$ we have $t_{1}: t_{\infty}>2: 3$. It follows that both $\mathcal{L}^{i}(t)$ and $\mathcal{F}^{i}(t)$ are contained in the corresponding sets for the previous choice of parameters $t_{\infty}(t)$ and $t_{1}(t)$. Hence, the convergence results remain true. For the new family, however, $\lim _{t \rightarrow 0}\left(t_{\infty}-t_{1}\right) / t_{\infty}=0$ holds. This implies that

$$
\lim _{i \rightarrow 0} \operatorname{aff}\left(\mathcal{C}_{j}^{i}, \mathcal{C}_{i}^{j}\right)=\left\{a f f\left(0_{\{i, j\}}, Q_{\{i, j\}}\right)\right\}
$$

Altogether, the $\mathcal{G}$-computation frame obtained satisfies, for small values of $t$, all the conditions of a regular one except the "unwished" collinearity of the points $\left\{Q_{\{i, j\}}\right\}_{i, j}$.

Now we start from such an "almost regular" $\mathcal{G}$-computation frame as constructed so far. We perform a perturbation which leaves all points unchanged except those ones depending on the position of $1^{i}$ in $\left(0^{i}, \infty^{i}\right)$. In particular, the points $0_{\{i, j\}}, \infty_{j}^{i}$, and $\infty_{i}^{j}$ are not changed. The position of $Q_{(i, j)}$ on aff $\left(\infty_{j}^{i}, \infty_{i}^{j}\right)$ depends strictly monotonically on the position of $1_{j}^{i} \in\left(0_{\{i, j\}}, \infty_{j}^{i}\right)$. In fact, the set of positions of $\left\{1_{j}^{i}\right\}_{i, j}$ for which there is no collinearity between points from $\left\{Q_{\{i, j\}}\right\}_{i, j}$ is open and dense. The position of each $1_{j}^{i}$ can easily be perturbed by a shifting of $1^{i} \in\left(0^{i}, \infty^{i}\right)$ with fixed $0^{i}, \infty^{i}$, and $Q_{j}^{i}$. The $\mathcal{G}$-computation frame can obviously be rebuilt such that the perturbation of any single point is arbitrarily small. This results in a regular $\mathcal{G}$-computation frame.

\section{Concluding Remarks}

In our proof we emphasized that a regular computation frame can be taken as a computation frame in general position. This yielded an easy argument to prove that the corresponding realization space $N=\mathcal{R}(\mathcal{N})$ is a smooth manifold. However, in this framework it is hard to analyze the topology of $N$ and to give an efficient computation of $\psi: \sigma \mapsto \mathcal{M}_{\sigma}$. If $\psi$ could be computed in polynomial time, then the problem of "stretchability" was proven to be at least as hard as the Existential Theory of the Reals (ETR). The latter asks whether a given algebraic variety is empty or not. The problem of stretchability is the question of realizability of an oriented matroid. It was proven by Shor [6] that stretchability is NP-hard.

Now it seems that we can introduce a constructive concept of a regular computation frame which supports the computation of $\psi$. The new concept also implies that $N$ can be taken in the form $\{1,-1\} \times \mathbb{R}^{l}$, where $\{1,-1\}$ stands for the choice of an orientation. Recall that this nice structure of $N$ has also been claimed by Mnëv [5]. In addition, for any sign-vector $\sigma$ the corresponding matroid $\mathcal{M}_{\sigma}$ can be computed in polynomial time in dependence on the maximal degree of the polynomials $f_{i}$ and the accumulated binary storage size required for their coefficients. Moreover, we can use one single 
(polynomial-time) algorithm for all input data $\left\{f_{1}, \ldots, f_{m}\right\} \subset \mathbb{Z}[X]$ and $\sigma \in\{-1,0,1\}^{m}$ for computation of

$$
\left(\left\{f_{1}, \ldots, f_{m}\right\}, \sigma\right) \mapsto \mathcal{M}_{\sigma}^{\left\{f_{1}, \ldots, f_{m}\right\}} .
$$

However, at present, all the details have not been worked out.

\section{Acknowledgments}

I would like to thank $H$. Th. Jongen for continuing support and encouragement. I am also grateful to R. Hirabayashi, N. Mnëv, J. Richter-Gerbert, M.-F. Roy, and G. M. Ziegler for stimulating discussion and to the referees for their valuable suggestions.

\section{References}

1. Björner, A., Las Vergnas, M., Sturmfels, B., White, N., and Ziegler, G. M., Oriented Matroids, Cambridge University Press, Cambridge, 1993.

2. Bokowski, J., and Sturmfels, B., Computational Synthetic Geometry, Lecture Notes in Mathematics, Vol. 1355, Springer-Verlag, Berlin, 1989.

3. Günzel, H., Hirabayashi, R., and Jongen, H. Th., Multiparametric optimization: on stable singularities occurring in combinatorial partition codes, Control and Cybernetics, 23, (1994), 153-167.

4. Mnëv, N. E., The universality theorems on the classification problem of configuration varieties and convex polytopes varieties, in Topology and Geometry-Rohlin Seminar, Viro, O. Y., ed., pp. 527-543, 1988.

5. Mnëv, N. E., The Universality Theorem on the Oriented Matroid Stratification of the Space of Real Matrices, DIMACS Series in Discrete Mathematics and Theoretical Computer Science, Vol. 6. American Mathematical Society, Providence, RI, 1991.

6. Shor, P. W., Stretchability of Pseudolines is NP-Hard (The Victor Klee Festschrift), DIMACS Series in Discrete Mathematics and Theoretical Computer Science, Vol. 4, 1991.

7. Young, J. W., Projective Geometry, The Open Court Publishing Company, Chicago, 1930.

Received June 22, 1994, and in revised form February 28, 1995. 